\title{
Decision Support Technology Research of Emergency Disposal $^{*}$
}

\author{
Qing Wang ${ }^{1,2 \#}$, Yuanchun Huang ${ }^{2}$, Yuelei He${ }^{2}$, Zhigang Liư ${ }^{2}$, Hua Hu${ }^{2}$, Aiqin Sun ${ }^{1,2}$ \\ ${ }^{1}$ The College of Business Administration, Shanghai University of Engineering Science, Shanghai, China; ${ }^{2}$ The College of Urban \\ Railway Transportation, Shanghai University of Engineering Science, Shanghai, China. \\ Email: ${ }^{\# 925558989 @ q q . c o m ~}$
}

Received November $12^{\text {th }}, 2013$; revised December $11^{\text {th }}, 2013$; accepted December $16^{\text {th }}, 2013$

Copyright (C) 2013 Qing Wang et al. This is an open access article distributed under the Creative Commons Attribution License, which permits unrestricted use, distribution, and reproduction in any medium, provided the original work is properly cited. In accordance of the Creative Commons Attribution License all Copyrights (C) 2013 are reserved for SCIRP and the owner of the intellectual property Qing Wang et al. All Copyright (c) 2013 are guarded by law and by SCIRP as a guardian.

\begin{abstract}
This paper focuses on the problem about how to efficiently process the emergency of rail transit and guarantee the lowest accident loss in a short period of time, which is the urban rail transit management policy that makers are faced with, and which develops a high integrated system with strong information based on contingency plans to give the decision aid of urban rail transit emergency events. The paper uses formal methods to present the emergency plan, generate the emergency disposal plan, meet the requirements of on-site emergency disposal, and it realizes the modernization of urban rail transit emergency management which has an important significance. Finally, taking a subway fire as an example, it describes the practicality of the auxiliary decision system.
\end{abstract}

Keywords: Emergency; Urban Rail Transit; Contingency Plans; Auxiliary Decision System

\section{Introduction}

With the expansion of rail transit network scale, requirements of emergency response system have also been improved. Sudden events are unpredictable and often cause great damage due to the inadequate preparation, which requires the staff to make the correct response in a very short period of time [1]. How to minimize the losses in a short time is the most important problem for management decision makers.

Emergency work flow is mainly about the accident information report and processing instructions issued. The paper makes the command center as the object, to establish the emergency disposal decision aid system.

\section{Key Support Technology Demand}

The core of emergency disposal of urban rail transit network emergencies is to enable decision makers to control the accident monitoring information of the scene, and

\footnotetext{
*This research was supported by Shanghai science and technology key project (Project No.: 11170501400), the People's Republic of China.

${ }^{\#}$ Corresponding author.
}

making use of the characteristic information of events according to the emergency response plan of the emergency disposal program dynamically generates solutions [2]. This article will focus on the following two aspects to research the emergency disposal of urban rail transit network aided decision support technology [3].

\subsection{Emergency Plans of Collaborative Integration Digital Model-Digital Model for Emergency Plan}

Multidimensional collaborative digital modeling of contingency plans refines plans to every process, every step, and comb out the relationship between various agencies, position, duties, personnel, process and resources, so that form an organic, interconnected and linkage of the overall [4].

The plan model of each dimension is not an isolated individual, but an interrelated, overall coordination. The relationship among the various dimensions is shown in Figure 1 [5]. According to the above model, the paper explains the plan to a multi-dimension, multi-perspective plan model. 


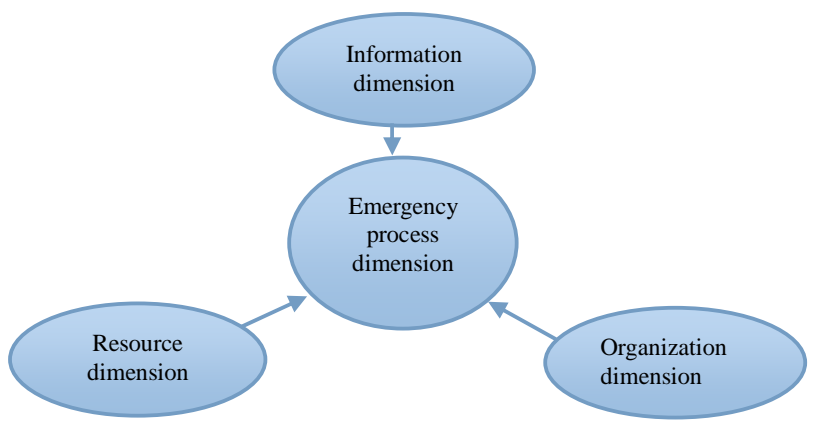

Figure 1. Emergency multidimensional integration model.

\subsection{Generation Technology Based on Plan}

The design idea of emergency disposal plan is as follows: 1) find a similar emergency event list feature information to match the sudden incident; 2) if the case library has storage solutions corresponding to the event, then extraction solution, and modification in proper way to form the eventual solution; 3) if the case base does not exist in the corresponding disposal scheme, using the number of emergency plan to find proper events [6]; 4) after acquire the solution, extract the handing steps to generate the final solution draft; 5) amendments by the user of the draft, and check the correctness of the modified, disposal the final plan; 6) send the plan to the scene, the scene of the accident deal with the accident according to the solution, disposal and real-time feedback accident, thereby circulating the process until the end to achieve dynamic adjustments to the disposal plan; 7) after the accident, the user should to judge whether the value of the emergency disposal plan, if valuable, store the emergency event to the relative case library to enrich the library [7]. In accordance with the above ideas, to achieve the above process, the first to solve the following two problems.

- Digital Alarm Emergencies: according to the accident and its influence degree and other basic information, automatic judging accident levels, providing disposal measures guiding the disposal of the accident scene to ensure the network safety operation, and automatically generate alarm record. Alarm template includes accident situation, accident level and alarm record. The accident situation mainly reflects the basic information of accident and the degree of influence [8]. Information form contains the occurrence time, duration, accident types, locations, detail place, location trips, casualties, expected running time, passenger flow and controllability to fully reflect the basic accident information, impact degree and scope. Accident level is used to reflect the degree of accident. According to the degree of harm, rail transit emergency may be caused by the propagation range, influence of size, the casualties and property losses, from high to low is divided into special major (grade
I), major (grade II), large (grade III), general (grade IV) .

- Improved Event Similarity Measure Model: in the field of CBR, there are kinds of event similarity algorithm based on the nearest neighbor algorithm; the nearest neighbor algorithm (K-Nearest Neighbor Algorithm, KNN) is the most commonly used [9]. Since KNN algorithm requires complete information of search condition, the paper introduces the structural similarity, local attribute similarity and attribute alternative conceptual on the basis of KNN algorithm to improve it [10]. The model is as follows. Assuming that matches the target event $\alpha$ and event $\beta, \alpha$ and $\beta$ are described by $m$ attributes,

$\alpha=\left\{a_{1}, a_{7}, a_{3}, \cdots, a_{m}\right\}, \quad \beta=\left\{b_{1}, b_{2}, b_{3}, \cdots, b_{m}\right\}$, and the attribute weights $w_{i}, i=1,2,3,4 \cdots, m$, calculation methods of similarity $\operatorname{SIM}(\alpha, \beta)$ between $\alpha$ and $\beta$ expressed as follows.

$$
\operatorname{SIM}(\alpha, \beta)=\omega_{\alpha, \beta}\left(1-\varphi_{\alpha, \beta}\right) \sum_{i=1}^{m} w_{i} \operatorname{sim}\left(a_{i}, b_{i}\right)
$$

The $\omega_{\alpha, \beta}$ means structural similarity between $\alpha$ and $\beta$, it describes the impact of missing data on event attributes similarity computing, $1-\varphi_{\alpha . \beta}$ represents the degree of substitutability between $\alpha$ and $\beta$, $\operatorname{sim}\left(a_{i}, b_{i}\right)$ means the local similarity in the properties of events between $\alpha$ and $\beta$.

1) The Computation of Similarity

The calculation process of structure similarity between target events and events are as follows. Assume that the target event set is $A_{n}$, all non empty attribute events set is $A_{1}, A_{0}$ and $A_{1}$ intersection marked $I$, and merger recorded as $U$; the weights of all attributes of set $I$ and $U$ respectively $\omega_{I}$ and $\omega_{U}$, and the definition of structural similarity between $\alpha$ and $\beta$ is as follows:

$$
\omega_{\alpha, \beta}=\frac{\omega_{I}}{\omega_{U}}
$$

\section{2) Calculation of Local Similarity}

The local similarity refers to events in each attribute similarity, emergency attribute value type can be divided into Continuous type, classification, fuzzy numbers or fuzzy interval types, different data types should have corresponding property local similarity measure. Symbolic attributes: in urban rail transit traffic accident, accident types, locations, the nature of the accident and other attributes are symbolic attributes. Their similarity is calculated as follows:

$$
\operatorname{sim}\left(a_{i}, b_{i}\right)= \begin{cases}1 & a_{i}=b_{i} \\ 0 & a_{i} \neq b_{i}\end{cases}
$$

The $a_{i}, b_{i}$ represents the property value between the target event $\alpha$ and $\beta$. 
Determined attribute similarity algorithm: the similarity method based on Hamming distance formula of evolution is calculated as follows.

$$
\operatorname{sim}\left(a_{i}, b_{i}\right)=1-\operatorname{dist}\left(a_{i}, b_{i}\right)=1-\frac{\left|a_{i}-b_{i}\right|}{\left|\max _{i}-\min _{i}\right|}
$$

The calculation method of fuzzy number type attribute similarity: fuzzy number default here is a convex fuzzy set. If $a_{i 1} \leq a_{i 2} \leq a_{i 3} \leq a_{i 4}$ and $b_{i 1} \leq b_{i 2} \leq b_{i 3} \leq b_{i 4}$, we can use Graded Mean Integration-representation Distance to calculate the similarity.

$$
\begin{aligned}
& \operatorname{sim}\left(a_{i}, b_{i}\right)=\left(1+\left|p\left(a_{i}\right)-p\left(b_{i}\right)\right|\right)^{-1} \\
& p\left(a_{i}\right)=\frac{a_{i 1}+2 a_{i 2}+2 a_{i 3}+a_{i 4}}{6} \\
& p\left(b_{i}\right)=\frac{b_{i 1}+2 b_{i 2}+2 b_{i 3}+b_{i 4}}{6}
\end{aligned}
$$

3) Calculate The Difference $\omega_{\alpha, \beta}$ Between The Target Event $\alpha$ and Event $\beta$

Variability of response is the difference between the degree of each unit in the overall difference, the greater, the more difficult to be replaced. It is calculated as follows.

$$
\begin{aligned}
\omega_{\alpha, \beta} & =\frac{\sigma_{\alpha, \beta}}{X_{\alpha, \beta}} \\
\bar{X}_{\alpha, \beta} & =\frac{1}{n} \sum_{i=1}^{m} \operatorname{sim}\left(a_{i}, b_{i}\right) \\
\sigma_{\alpha, \beta} & =\frac{1}{n} \sqrt{\sum_{i=1}^{m} w_{i}\left[\operatorname{sim}\left(a_{i}, b_{i}\right)-\bar{X}_{\alpha, \beta}\right]^{2}}
\end{aligned}
$$

4) Calculation of Event Feature Attribute Weight

This paper uses the attribute hierarchy model (Attribute Hierarchical Model AHM) [11] to calculate the weight of the event's attribute value. AHM is a method to calculate the relative weight of an attribute, the attribute weights can be adapted to the calculation in the absence of input information, to meet the weight loss or error the operator filled in information of computing attribute in emergency situation.

The AHM method is based on the improved analytic hierarchy process AHP (Analytic Hierarchy Process), according to the Table 1 show, mode on the relative importance of each attribute was scoring assessment by experts in rail transit safety.

Table 1. Attribute important degree evaluation.

\begin{tabular}{ccccccc}
\hline $\begin{array}{c}\mathrm{x} \text { is } \begin{array}{c}* \text { important } \\
\text { than } \mathrm{y}\end{array} \\
\text { Fraction }\end{array}$ & $\underline{\text { equally }}$ & $\underline{\underline{\text { slightly }}}$ & $\underline{\text { more }}$ & $\frac{\underline{\text { much }}}{\text { more }}$ & $\underline{\underline{\text { very much }}}$ \\
\hline
\end{tabular}

After require the relative importance $a_{i i}$ between attributes $a_{i}$ and $a_{i}$, the property transfers $u_{i i}$ between the important degrees were calculated by formula (7). The transfer of property represents the important degree of attributes $a_{i}$ and $a_{j}$. $i=1,2,3, \cdots, m ; j=1,2,3, \cdots, m$.

$$
u_{i j}= \begin{cases}0, & a_{i j}=1, i=j \\ 0.5, & a_{i j}=1, i \neq j \\ \frac{1}{2 k+1}, & a_{i j}=\frac{1}{k} \\ \frac{2 k}{2 k+1}, & a_{i j}=k\end{cases}
$$

Transfer important degree was calculated by the use of formula (8). The weight of each attribute $w_{i j} n$ means the number of attributes of the event $C$.

$$
\omega_{i}=\frac{2}{n(n-1)} \sum_{j=1}^{n} u_{i j}
$$

\section{Implementation of Emergency Aided Decision Support Technology}

\subsection{Digital Template Emergency Plan to Take Station Fire Plan as an Example}

The emergency plan of city subway station fire accident is modeled by the emergency plan of multidimensional digital model proposed in the previous paper, and established digital template. The template also includes five dimensions of organizational, resource, process, function and information. Table 2 shows the metro station fire accident contingency plan digital template, since the original template format is too wide to show completely in this page, here just intercepted a part template.

\subsection{Emergency Case}

The following emergency treatment takes a subway fire accident as an example, to introduce the process of decision making by using the technology of emergency disposal. The background for a car fire smoke, informed the station integrated control, integrated control officer to report the line dispatching immediately; another train reach to the station platform, the fourth compartment fire can't be controlled, 7 passengers suffered serious burns, the comprehensive control center of the line command station buckle car, which is expected to break 1 - $4 \mathrm{~h}$, and report to the command center, and report to the command center, launched the emergency plan.

- Event Alarm: After the occurrence of unexpected events, information station integrated control officer reported in the alarm interface according to the situation of emergency, and share its to the OCC, enterprises total harmonic command center TCC. 
Table 2. Fire emergency plan digital template (part).

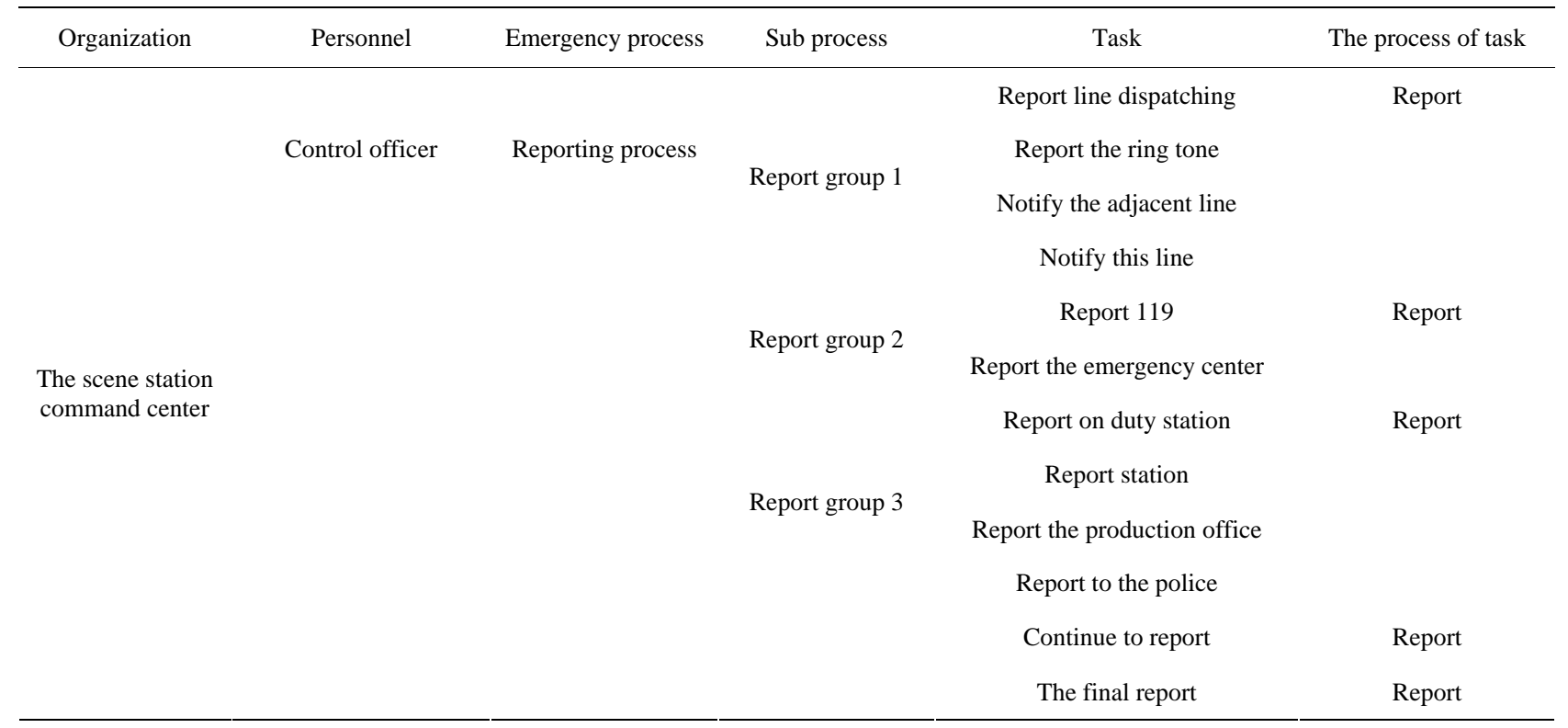

Table 3. Event attribute.

\begin{tabular}{|c|c|c|c|c|c|}
\hline The property & $C_{1}$ & $C_{2}$ & $C_{3}$ & $C_{4}$ & $C_{5}$ \\
\hline Accident type $A_{1}$ & Transfer station & Non transfer station & Transfer station & Section & Transfer station \\
\hline The location $A_{2}$ & A & B & $\mathrm{C}$ & $\mathrm{D}$ & E \\
\hline Accident properties $A_{3}$ & Fire & Flood & Large passenger & Catenaries power outages & $\begin{array}{l}\text { Extraterrestrial } \\
\text { Injury }\end{array}$ \\
\hline The specific location $A_{4}$ & Orbit range & Near the railway & $N / A$ & Orbit range & Orbit range \\
\hline Happened time $A_{5}$ & $16: 20$ & $14: 20$ & $07: 50$ & $18: 15$ & $08: 25$ \\
\hline Accidents level $A_{6}$ & II & II & III & III & IV \\
\hline The number of casualties $A_{7}$ & 2 & $N / A$ & $N / A$ & 1 & 1 \\
\hline Interruption of operation time $A_{8}$ & $0.5-1 \mathrm{~h}$ & $3-10 h$ & $1-3 h$ & $1-3 h$ & $0-0.5 \mathrm{~h}$ \\
\hline Passenger flow $A_{9}$ & Large & Small & Large & Large & Medium \\
\hline Controllability $A_{10}$ & Strong & Weak & Medium & Medium & Strong \\
\hline
\end{tabular}

- Matching Emergency Plan: According to the alarm information, get the target event, its attributes: \{accident, a transfer station, fire, train accident, 17:35, grade II, 7, 1 - $4 \mathrm{~h}$, the big, strong\}. Calculate the event similarity, as shown in Table 3. First of all, using the above formula to calculate the weight of each attribute of the event, as shown in Table 4; and then one by one to calculate structural similarity, event attribute similarity and different local event property substitutability between the settlement results as shown in Table 5.

According to the calculation result of the global similarity by formula (1), the similarity of event $C_{1}$ and target event is 0.738507 . Define the event $C_{1}$ and target event to match most, at the same time, to search for the corresponding emergency plan for the digital modeling, and get digital template contingency plans such as shown in Table 2.

According to the login information, system can require attention to the relevance information in the emergency. This will ensure that the disposal personnel can get the information they need in the process to dispose of accidents, improve the efficiency of emergency. Through the above process, we found that the decision by the emergency disposal technology can effectively ensure all levels of staff to get their goal in the shortest time, improve efficiency, reduce the reaction time, and contribute to the minimum loss of the accident, so that the rail transit sys- 
Table 4. Attribute weights calculation.

\begin{tabular}{ccccccccccccc}
\hline Property & $A_{1}$ & $A_{2}$ & $A_{3}$ & $A_{4}$ & $A_{5}$ & $A_{6}$ & $A_{7}$ & $A_{8}$ & $A_{9}$ & $A_{10}$ & Weight \\
\hline$A_{1}$ & 0 & 0.70 & 0.20 & 0.50 & 0.70 & 0.30 & 0.30 & 0.25 & 0.50 & 0.20 & 0.118889 \\
$A_{2}$ & 0.30 & 0 & 0.10 & 0.20 & 0.50 & 0.10 & 0.10 & 0.15 & 0.15 & 0.10 & 0.162222 \\
$A_{3}$ & 0.80 & 0.90 & 0 & 0.90 & 0.90 & 0.30 & 0.75 & 0.75 & 0.80 & 0.70 & 0.048889 \\
$A_{4}$ & 0.50 & 0.80 & 0.10 & 0 & 0.90 & 0.30 & 0.75 & 0.75 & 0.80 & 0.70 & 0.117778 \\
$A_{5}$ & 0.30 & 0.50 & 0.10 & 0.20 & 0 & 0.10 & 0.10 & 0.10 & 0.20 & 0.10 & 0.164444 \\
$A_{6}$ & 0.70 & 0.90 & 0.70 & 0.80 & 0.90 & 0 & 0.70 & 0.70 & 0.80 & 0.50 & 0.053333 \\
$A_{7}$ & 0.70 & 0.90 & 0.25 & 0.70 & 0.90 & 0.30 & 0 & 1 & 0.75 & 0.30 & 0.092222 \\
$A_{8}$ & 0.75 & 0.85 & 0.25 & 0.70 & 0.90 & 0.30 & 0.50 & 0 & 0.70 & 0.30 & 0.093333 \\
$A_{9}$ & 0.50 & 0.85 & 0.20 & 0.50 & 0.80 & 0.20 & 0.25 & 0.30 & 0 & 0.20 & 0.122222 \\
$A_{10}$ & 0.80 & 0.90 & 0.30 & 0.80 & 0.90 & 0.50 & 0.70 & 0.70 & 0.80 & 0 & 0.068889 \\
\hline
\end{tabular}

Table 5. Calculation results of event and target event similarity.

\begin{tabular}{|c|c|c|c|c|c|c|}
\hline The attribute of local similarity & $C_{1}$ & $C_{2}$ & $C_{3}$ & $C_{4}$ & $C_{5}$ & Weight \\
\hline$A_{1}$ & 1 & 0 & 1 & 0 & 1 & 0.124628 \\
\hline$A_{2}$ & 1 & 0 & 0 & 0 & 0 & 0.102512 \\
\hline$A_{3}$ & 1 & 0 & 0 & 0 & 0 & 0.063865 \\
\hline$A_{4}$ & 0 & 0 & $N / A$ & 1 & 1 & 0.124582 \\
\hline$A_{5}$ & 0.845 & 0.932 & 0.568 & 0.954 & 0.631 & 0.118135 \\
\hline$A_{6}$ & 1 & 1 & 0.75 & 0.75 & 0.25 & 0.069671 \\
\hline$A_{7}$ & 0.135 & $N / A$ & $N / A$ & $N / A$ & 0.135 & 0.120473 \\
\hline$A_{8}$ & 0.425 & 0.322 & 1 & 1 & 0.387 & 0.121925 \\
\hline$A_{9}$ & 1 & 0 & 1 & 1 & 0 & 0.064216 \\
\hline$A_{10}$ & 1 & 0 & 0 & 0 & 1 & 0.089992 \\
\hline Property substitutability & 0.959851 & 0.960240 & 0.952419 & 0.899305 & 0.953287 & \\
\hline Structural similarity & 1 & 0.896456 & 0.802031 & 0.869856 & 1 & \\
\hline Event similarity & 0.738507 & 0.178978 & 0.310633 & 0.319864 & 0.391386 & \\
\hline
\end{tabular}

tem can return to normal operation as soon as possible.

\section{Conclusion and Research Prospect}

This paper analyzes the characteristics of Chinese urban rail transit network operation and designs road network emergency response decision support system. Meanwhile, the key techniques involved are studied; the emergency command system architecture under the condition of network operation of urban rail transit is proposed; the emergency disposal work flow in this command system through the investigation is deduced.

\section{REFERENCES}

[1] Z. Z. Wu and M. Liu, "Major Accident Emergency Rescue System and Plan the Introduction,” Metallurgical Industry Press, Beijing, 2003, pp. 1-5.

[2] "National Disposal of City Subway Accident Disaster Emergency Plan,” Policies and Documents, The State Council Issued in January 8, 2006.

[3] Y. P. Cui, Z. M. Tang and X. Wu, "System Research. Emergency Handling of Metro Accidents Based on Multi-Agent," Journal of the China Railway Society, Vol. 26, No. 3, 2004, pp. 8-12. 
[4] W. J. Li, Y. Qin and L. M. Jia, "Design and Implementation of Gateway of City Railway Transportation Emergency Management System,” 2007 3rd China Intelligent Transports Annual Conference, Nanjing, 15 December 2007, pp. 67-97.

[5] H. S. Zhu, "Thinking of Shanghai City Rail Transit Network Operation Management," Modern City Rail Transportation, Vol. 4, 2007, pp. 10-13.

[6] Z. Q. Wang, M. Zhang and H. Xu, "Emergency Treatment Assistant Decision System of Urban Rail Transit," International Conference on Transportation Engineering, Vol. 14, 2007, pp. 3707-3712.

[7] Y. J. He, “Individual Business Process Model,” Journal of Shandong University, 2008.

[8] "Digital Plan Template and Template Digital Alarm Information Report,” Beijing Jiaotong University, Beijing,
2012.

[9] S. J. Gu, X. D. Gao and R. Sun, “An Improved CBR Case Retrieval Similarity Measure Model,” Chinese Journal of Management Information, Vol. 14, No. 9, 2011, pp. 5055.

[10] Z. L. Liao, X. W. Mao, Y. H. Liu, Z. X. Xu and P. M. Hannam, "CBR Respond and Preparedness System Development for Environmental Emergency,” Civil Engineering and Environmental Systems, Vol. 28, No. 4, 2011, pp. 301-323.

http://dx.doi.org/10.1080/10286608.2011.604416

[11] D. Wettschereck, D. W. Aha and T. Mohri, “A Review and Empirical Evaluation of Feature Weighting Methods for a Class of Lazy Learning Algorithms," Artificial Intelligence Review, Vol. 11, No. 1-5, 1997, pp. 273-314. 